\title{
Interleukin-2 and Interleukin-8 Gene Polymorphisms and Acquired Aplastic Anemia Risk in a Chinese Population
}

\author{
Xuejie Zhang ${ }^{a}$ Shengyun Lin ${ }^{b}$ Yan Yang ${ }^{c}$ Liucheng Rong ${ }^{a}$ Guangsheng He ${ }^{d}$ \\ Hailong $\mathrm{He}^{\mathrm{e}}$ Yao Xue ${ }^{\mathrm{a}}$ Yaping Wang ${ }^{\text {a }}$ Yongjun Fang ${ }^{\mathrm{a}}$ \\ aDepartment of Hematology and Oncology, Children's Hospital of Nanjing Medical University, Nanjing, \\ 'Department of Hematology, The First Affiliated Hospital, Zhejiang Chinese Medical University, \\ Zhejiang, 'Department of Hematology, The First Hospital of Jilin University, Jilin, dDepartment of \\ Hematology, The First Affiliated Hospital of Nanjing Medical University, Nanjing, eDepartment of \\ Hematology, The Affiliated Soochow Children's Hospital of Soochow University, Suzhou, China
}

\section{Key Words}

Acquired aplastic anemia • Interleukin-2 • Interleukin-8 • Polymorphism • Transcription

\begin{abstract}
Background/Aims: Cytokines IL-2 and IL-8 both participate in immune regulation. However, the relationship between polymorphisms in these two cytokines and the risk of acquired aplastic anemia (acquired AA) has not been explored. Methods: We selected five SNPs including rs11575812, rs2069772 and rs2069762 of IL-2, rs2227306 and rs2227543 of IL-8. SNaPshot genotyping was used to test the genotypes of IL-2 and IL-8 polymorphisms in a population of 101 acquired AA patients and 165 healthy controls. Results: The rs 2069762 G allele appeared to be a protective mutation, but no significant differences were found in other four SNPs. We also found that rs2069762 had an impact on the transcriptional regulation. Conclusions: It could be assumed that the rs 2069762 polymorphism might reduce the risk of acquired aplastic anemia, while the remaining four SNPs might not contribute to susceptibility to acquired $A A$ in a Chinese population.

\section{Introduction}

Acquired aplastic anemia (AA) is a rare hematologic disease characterized by pancytopenia in peripheral blood (PB) and bone marrow (BM) hypoplasia [1]. The main clinical manifestations of acquired AA patients are anemia, hemorrhage and infection [2]. Although the pathogenesis of acquired AA is complicated and has not been fully understood, the immune-mediated destruction of hematopoietic stem cells (HSCs) is believed to be the main nosogenesis [3]. Recent studies suggest that aberrant $\mathrm{T}$ cell immune homeostasis plays 
a key role in the pathogenesis of acquired AA [4]. Abnormal activation of Th1 T-cell causes excessive production of hematopoietic negative regulatory cytokines, such as interleukin-2 (IL-2), interferon- $\gamma$ (IFN- $\gamma$ ), IL-8, tumor necrosis factor- $\alpha$ (TNF- $\alpha$ ) and so on. These cytokines contribute to inhibiting hematopoiesis, which is correlated with disease activity $[5,6]$.

As an important immunoregulatory cytokine, IL-2 is a autocrine and paracrine molecule, which is produced mainly by activated CD4+ T-cells, naive CD8+ T-cells and dendritic cells [7]. It was reported that IL-2 could stimulate the activation, proliferation and differentiation of many immune cells including natural killer (NK), B and T-cells [8]. So, as a T-cell growth factor, IL-2 is mainly produced by active T cells and in turn affects the proliferation of T-cells. IL-2 has pleiotropic effects on the regulatory function of immune system [9]. Another hematopoietic negative regulatory factor, Interleukin-8, is an angiogenic chemokine, which can be produced by different cell types, such as neutrophils, macrophages, epithelial cells and endothelial cells [10]. Interleukin-8 is an effective inhibitor of myelopoiesis [6]. According to previous studies, levels of IL-2 and IL- 8 were both evidently increased in the bone marrow and peripheral blood of acquired AA patients when compared to their healthy controls. So we propose that the abnormality of these two cytokines might cause immune dysfunction and lead to the suppression of haemopoiesis, resulting in the occurrence of acquired aplastic anemia $[6,11]$.

It is well known that single nucleotide polymorphisms (SNPs) may influence the individual susceptibility to diseases. Polymorphic variants in IL-2 region have already been discovered to be related to a number of autoimmune diseases, such as type 1 diabetes (T1D) [12], rheumatoid and juvenile idiopathic arthritis [13,14], multiple sclerosis [15], psoriasis [16], inflammatory bowel disease and systemic lupus erythematosus (SLE) [17-19]. In addition, some previous studies have revealed that the IL-8 gene polymorphisms are linked to higher risk of osteoarthritis, osteosarcoma, oral and gastric cancer [20-23]. However, little was known about the association between these two cytokine genes polymorphisms and the risk of acquired AA.

In our study, we selected the SNPs whose minor allele frequency (MAF) value were greater than $5 \%$ in the Chinese population and located in potential functional regions of IL-2 and IL-8. According to these criteria, we identified three SNPs of IL-2 (rs11575812, rs2069772 and rs2069762) and two SNPs of IL-8 (rs2227306 and rs2227543) using Pubmed and the Hapmap database. Besides, the rs2069762 in the IL-2 promoter, which had already been researched in other autoimmune diseases studies [12-19], was also included in our study. Consequently, a case-control study was performed to evaluate the relevance between these five SNPs and the risk of acquired AA in a Chinese population.

\section{Materials and Methods}

\section{Study subject}

Our study subjects contain 101 acquired AA patients and 165 healthy controls, who were recruited from Children's Hospital of Nanjing Medical University, the First Affiliated Hospital of Nanjing Medical University, the Affiliated Soochow Children's Hospital of Soochow University, the First Hospital of Jilin University and the First Affiliated Hospital, Zhejiang Chinese Medical University from July 2014 to 2015 November. The mean age $( \pm$ SD) of these cases was $24.7 \pm 21.1$ years. Patients were diagnosed with acquired AA by biopsy and peripheral blood (PB) cell count. Their PB cell count involved at least two lineages, values have to lower than $<100 \mathrm{~g} / \mathrm{L}$ for hemoglobin, than $1.5 \times 10^{9} / \mathrm{L}$ for neutrophils and than $50 \times 10^{9} / \mathrm{L}$ for platelets. Bone marrow presented with reduced hematopoietic cells while major dysplastic signs and marrow fibrosis were ruled out. Combined with detailed physical examination, radiography of bone and related gene detection, we excluded Fanconi anemia and dyskeratosis congenital. These controls, with a mean age $( \pm S D)$ of $27.2 \pm 21.2$ years, had no blood disease or cancer history. All controls were matched to cases in terms of gender and age ( \pm 16 years) and have no genetic relationship with cases. Our research program was approved by the Institutional Review Board of Children's Hospital of Nanjing Medical University.

\section{KARGER}


Genotyping

Genomic DNA was extracted from peripheral blood of every study subject by using the standard protocols of Relax Gene Blood DNA System (TIANGEN, Beijing, China). Blood samples of patients were all collected before clinical treatments. The DNA samples were stored at $-20^{\circ} \mathrm{C}$ until used. Five SNPs were detected in the DNA samples from 101 cases and 165 controls by using the method of SNaPshot.

Statistical analysis

To identify possible selection bias and genotyping errors, Hardy-Weinberg equilibrium of the genotype distribution among control subjects was estimated using a goodness of fit $\chi 2$ test. The crude and adjusted odds ratios (OR) for risk of acquired AA and 95\% confidence intervals (CI) were calculated by univariate and multivariate logistic regression analyses. The multivariate adjustment included age $( \pm 16$ years) and gender. All tests were two-sided and P-value $<0.05$ was regarded as statistically significant. Statistical analyses were carried out by Statistical Analysis System software (version 9.2; SAS Institute, Cary, NC, USA).

Functional prediction

Bioinformatics tools of SNPinfo (http://snpinfo.niehs.nih.gov/snpinfo/snpfunc.php) and F-SNP (http://compbio.cs.queensu.ca/F-SNP/) were used to predict the potential functions of significant SNPs.

\section{Results}

Correlation analysis results

The frequency distributions of the selected demographic variables of acquired AA patients and controls are shown in Table 1 . There were no statistical differences in age $(\mathrm{P}=0.502)$ and sex $(\mathrm{P}=0.441)$ between the case and control groups.

Table 2 showed the primary information and allele frequencies of these selected SNPs. The allele frequencies of these five polymorphisms conformed to Hardy-Weinberg equilibrium among the control group $(0.790,0.624,0.258,0.394$ and 0.423 , respectively). Allele analysis of rs2069762 revealed different distributions between cases and controls $(\mathrm{P}=0.022)$.

The genotypic distributions of the selected five SNPs are summarized in Table 3. In general, one SNP rs2069762 demonstrated different distributions between the case and

Table 1. Frequency distributions of age and gender in Chinese acquired AA cases and controls.*Two-sided chi-square test for frequency distributions of age and gender between cases and controls

\begin{tabular}{|c|c|c|c|c|c|}
\hline \multirow{2}{*}{ Variables } & \multicolumn{2}{|c|}{ Cases $(n=101)$} & \multicolumn{2}{|c|}{ Controls $(\mathrm{n}=165)$} & \multirow{2}{*}{$\mathrm{P}^{*}$} \\
\hline & $\mathrm{n}$ & $\%$ & $\mathrm{n}$ & $\%$ & \\
\hline \multicolumn{6}{|c|}{ Age (years) } \\
\hline$\leqq 16$ & 44 & 43.56 & 64 & 38.79 & 0.502 \\
\hline$>16$ & 57 & 56.44 & 101 & 61.21 & \\
\hline \multicolumn{6}{|l|}{ Gender } \\
\hline Male & 57 & 56.44 & 100 & 60.61 & 0.441 \\
\hline Female & 44 & 43.56 & 65 & 39.39 & \\
\hline
\end{tabular}

Table 2.Primary information of genotyped SNPs. ${ }^{a}$ Two-sided $\chi^{2}$ test for allele frequencies between cases and controls. ${ }^{b}$ value for Hardy-Weinberg equilibrium in the control group

\begin{tabular}{lllllllll}
\hline SNP & Base change & Gene name & Chr & \multicolumn{3}{c}{ Minor allele frequencies } & Pa & Hardy-Weinberg \\
& & & & Database & cases & controls & & equilibriumb \\
rs11575812 & T $>$ C & IL-2 & 4 & 0.102 & 0.114 & 0.088 & 0.327 & 0.790 \\
rs2069772 & A $>$ G & IL-2 & 4 & 0.105 & 0.109 & 0.096 & 0.658 & 0.624 \\
rs2069762 & T>G & IL-2 & 4 & 0.239 & 0.287 & 0.385 & $\mathbf{0 . 0 2 2}$ & 0.258 \\
rs2227306 & C >T & IL-8 & 4 & 0.367 & 0.366 & 0.370 & 0.938 & 0.394 \\
rs2227543 & C>T & IL-8 & 4 & 0.379 & 0.391 & 0.385 & 0.886 & 0.423 \\
\hline
\end{tabular}


Table 3. Logistic regression analysis was used to evaluate the connection between cytokines polymorphis$\mathrm{ms}$ and acquired AA risk. ${ }^{\dagger}$ The $\mathrm{ORs}, 95 \% \mathrm{CIs}$ and $\mathrm{P}$ value were calculated after adjusting for age and gender

\begin{tabular}{|c|c|c|c|c|c|c|c|}
\hline \multirow[b]{2}{*}{ Genotype } & \multicolumn{2}{|c|}{$\begin{array}{l}\text { Cases } \\
(\mathrm{n}=101)\end{array}$} & \multicolumn{2}{|c|}{$\begin{array}{l}\text { Controls } \\
(\mathrm{n}=165)\end{array}$} & \multirow[t]{2}{*}{ OR( $(95 \% \mathrm{CI})$} & \multirow[t]{2}{*}{ Adjusted OR $(95 \% \mathrm{CI})^{\dagger}$} & \multirow[t]{2}{*}{ Adjusted P value } \\
\hline & n & $\%$ & $\mathrm{n}$ & $\%$ & & & \\
\hline rs1157582 & $\mathrm{T} / \mathrm{C}$ & & & & & & \\
\hline TT & 80 & 79.21 & 137 & 83.03 & 1.00 (reference) & $1.00($ reference $)$ & \\
\hline CT & 19 & 18.81 & 27 & 16.36 & $1.21(0.63-2.31)$ & $1.2(0.63-2.35)$ & 0.555 \\
\hline $\mathrm{CC}$ & 2 & 1.98 & 1 & 0.61 & $3.42(0.30-38.36)$ & $3.69(0.33-41.82)$ & 0.291 \\
\hline $\mathrm{CC}+\mathrm{CT}$ & 21 & 20.79 & 28 & 16.97 & $1.28(0.68-2.41)$ & $1.31(0.69-2.47)$ & 0.408 \\
\hline $\mathrm{TT}+\mathrm{CT}$ & 99 & 98.02 & 164 & 99.39 & $1.00($ reference $)$ & $1.00($ reference $)$ & \\
\hline $\mathrm{CC}$ & 2 & 1.98 & 1 & 0.61 & $3.31(0.30-37.00)$ & $3.59(0.32-40.53)$ & 0.302 \\
\hline rs2069772 & $\mathrm{A} / \mathrm{G}$ & & & & & & \\
\hline $\mathrm{AA}$ & 82 & 81.19 & 134 & 81.21 & 1.00 (reference) & 1.00 (reference) & \\
\hline$A G$ & 16 & 15.84 & 30 & 18.18 & $0.87(0.45-1.70)$ & $0.84(0.43-1.65)$ & 0.613 \\
\hline GG & 3 & 2.97 & 1 & 0.61 & $4.90(0.50-47.91)$ & $5.79(0.58-58.09)$ & 0.136 \\
\hline $\mathrm{GG}+\mathrm{AG}$ & 19 & 18.81 & 31 & 18.79 & $1.00(0.53-1.89)$ & $0.98(0.52-1.86)$ & 0.961 \\
\hline $\mathrm{AA}+\mathrm{AG}$ & 98 & 97.03 & 164 & 99.39 & 1.00 (reference) & 1.00 (reference) & \\
\hline GG & 3 & 2.97 & 1 & 0.61 & $5.02(0.52-48.92)$ & $5.94(0.59-59.47)$ & 0.130 \\
\hline rs2069762 & $\mathrm{T} / \mathrm{G}$ & & & & & & \\
\hline TT & 51 & 50.50 & 59 & 35.76 & 1.00 (reference) & 1.00 (reference) & \\
\hline GT & 42 & 41.58 & 85 & 51.52 & $0.57(0.34-0.97)$ & $0.56(0.33-0.95)$ & 0.030 \\
\hline GG & 8 & 7.92 & 21 & 12.73 & $0.44(0.18-1.08)$ & $0.46(0.19-1.13)$ & 0.089 \\
\hline $\mathrm{GG}+\mathrm{GT}$ & 50 & 49.50 & 106 & 64.24 & $0.55(0.33-0.90)$ & $0.54(0.32-0.89)$ & 0.016 \\
\hline $\mathrm{TT}+\mathrm{GT}$ & 93 & 92.08 & 144 & 87.27 & 1.00 (reference) & 1.00 (reference) & \\
\hline GG & 8 & 7.92 & 21 & 12.73 & $0.59(0.25-1.39)$ & $0.61(0.26-1.45)$ & 0.267 \\
\hline Rs2227306 & $\mathrm{C} / \mathrm{T}$ & & & & & & \\
\hline $\mathrm{CC}$ & 41 & 40.59 & 63 & 38.18 & 1.00 (reference) & 1.00 (reference) & \\
\hline $\mathrm{CT}$ & 46 & 45.54 & 82 & 49.70 & $0.86(0.51-1.47)$ & $0.86(0.51-1.48)$ & 0.593 \\
\hline TT & 14 & 13.86 & 20 & 12.12 & $1.07(0.49-2.37)$ & $1.06(0.48-2.33)$ & 0.889 \\
\hline $\mathrm{TT}+\mathrm{CT}$ & 60 & 59.41 & 102 & 61.82 & $0.90(0.55-1.50)$ & $0.90(0.54-1.50)$ & 0.693 \\
\hline $\mathrm{CC}+\mathrm{CT}$ & 87 & 86.14 & 145 & 87.88 & 1.00 (reference) & 1.00 (reference) & \\
\hline TT & 14 & 13.86 & 20 & 12.12 & $1.17(0.56-2.43)$ & $1.147(0.55-2.39)$ & 0.717 \\
\hline Rs 2227543 & $\mathrm{C} / \mathrm{T}$ & & & & & & \\
\hline $\mathrm{CC}$ & 38 & 37.62 & 60 & 36.36 & 1.00 (reference) & 1.00 (reference) & \\
\hline CT & 47 & 46.53 & 83 & 50.30 & $0.89(0.52-1.54)$ & $0.90(0.52-1.55)$ & 0.705 \\
\hline TT & 16 & 15.84 & 22 & 13.33 & $1.15(0.54-2.46)$ & $1.13(0.52-2.42)$ & 0.761 \\
\hline $\mathrm{TT}+\mathrm{CT}$ & 63 & 62.38 & 105 & 63.64 & $0.95(0.57-1.58)$ & $0.95(0.57-1.59)$ & 0.840 \\
\hline $\mathrm{CC}+\mathrm{CT}$ & 85 & 84.16 & 143 & 86.67 & 1.00 (reference) & 1.00 (reference) & \\
\hline TT & 16 & 15.84 & 22 & 13.33 & $1.22(0.61-2.46)$ & $1.19(0.59-2.41)$ & 0.620 \\
\hline
\end{tabular}

Table 4. Association between rs2069762 polymorphism and selected demographic data. \#The ORs and 95\% CIs were calculated after adjusting for age and gender

\begin{tabular}{lllllllll}
\hline \multicolumn{2}{c}{ Case } & Control & & \multicolumn{5}{c}{ \#Adjusted OR(95\% Cl) } \\
& $\mathrm{n}(\%)$ & $\mathrm{n}(\%)$ & $\mathrm{TT}$ & $\mathrm{GT}$ & $\mathrm{GG}$ & $\mathrm{GT} / \mathrm{GG}$ & $\mathrm{TT} / \mathrm{GT}$ & $\mathrm{GG}$ \\
\hline Sex & & & & & & & & \\
Male & $57(56.44)$ & $100(60.61)$ & 1.00 (reference) & $0.55(0.27-1.12)$ & $0.55(0.20-1.51)$ & $0.55(0.29-1.07)$ & 1.00 (reference) & $0.73(0.28-1.90)$ \\
Female & $44(43.56)$ & $65(39.39)$ & 1.00 (reference) & $0.56(0.25-1.24)$ & $0.21(0.02-2.01)$ & $0.52(0.24-1.14)$ & 1.00 (reference) & $0.30(0.03-2.68)$ \\
Age & & & & & & & & \\
$<=16$ & $44(43.56)$ & $64(38.79)$ & 1.00 (reference) & $0.77(0.34-1.74)$ & $0.48(0.11-2.12)$ & $0.71(0.32-1.55)$ & 1.00 (reference) & $0.55(0.13-2.28)$ \\
$>16$ & $57(56.44)$ & $101(61.21)$ & 1.00 (reference) & $\mathbf{0 . 4 4 ( 0 . 2 2 - 0 . 8 9 )}$ & $0.44(0.14-1.37)$ & $\mathbf{0 . 4 4 ( 0 . 2 3 - 0 . 8 6 )}$ & 1.00 (reference) & $0.66(0.22-1.95)$ \\
\hline
\end{tabular}

control groups, no significant differences were found in other four SNPs. The rs2069762 G allele appeared to be a protective mutation. According to the results of analysis of rs2069762, the GT (OR=0.56, 95\% $\mathrm{CI}=0.33-0.95, \mathrm{P}=0.030)$ and $\mathrm{GT} / \mathrm{GG} \quad(\mathrm{OR}=0.54,95 \% \mathrm{CI}=0.32$ $0.89, \mathrm{P}=0.016)$ genotypes showed reduced risk of acquired $\mathrm{AA}$, while the GG $(\mathrm{OR}=0.46$, $95 \% \mathrm{CI}=0.19-1.13, \mathrm{P}=0.089$ ) genotype showed no statistical significance. 
Zhang et al.: IL-2 and IL-8 Polymorphisms with Acquired AA

Table 5. Comparisons of distributions of the genotypes of five SNPs between NSAA, vSAA and SAA, patients and the controls, which used the logistic regression analysis. *Adjusted for age and gender. Eight patients lack the information of disease severity

\begin{tabular}{|c|c|c|c|c|c|c|c|c|c|c|c|c|}
\hline \multirow{3}{*}{$\begin{array}{l}\text { Genotype } \\
\text { rs11575812 }\end{array}$} & \multirow{2}{*}{\multicolumn{2}{|c|}{$\begin{array}{l}\text { Controls } \\
n=165\end{array}$}} & \multicolumn{4}{|c|}{ Disease severity } & \multicolumn{2}{|c|}{ OR $(95 \% \mathrm{CI})$} & \multicolumn{2}{|c|}{ *Adjusted OR (95\% CI ) } & \multicolumn{2}{|c|}{$\begin{array}{l}\text { *Adjusted } \\
\text { P value }\end{array}$} \\
\hline & & & \multicolumn{2}{|c|}{$\begin{array}{l}\text { NSAA } \\
\mathrm{n}=24\end{array}$} & \multicolumn{2}{|c|}{$\begin{array}{c}S A A+v S A A \\
n=69\end{array}$} & \multirow[t]{2}{*}{ NSAA } & \multirow[t]{2}{*}{$S A A+V S A A$} & \multirow[t]{2}{*}{ NSAA } & \multirow[t]{2}{*}{$S A A+v S A A$} & \multirow[t]{2}{*}{ NSAA } & \multirow[t]{2}{*}{$\mathrm{SAA}+\mathrm{VSAA}$} \\
\hline & $\mathrm{n}$ & $\%$ & $\mathrm{n}$ & $\%$ & $\mathrm{n}$ & $\%$ & & & & & & \\
\hline TT & 137 & 83.03 & 17 & 70.83 & 56 & 81.16 & $\begin{array}{l}1.00 \\
\text { (reference) }\end{array}$ & $\begin{array}{l}1.00 \\
\text { (reference) }\end{array}$ & $\begin{array}{l}1.00 \\
\text { (reference) }\end{array}$ & $\begin{array}{l}1.00 \\
\text { (reference) }\end{array}$ & & \\
\hline CT & 27 & 16.36 & 6 & 25.00 & 13 & 18.84 & $\begin{array}{l}1.79 \\
(0.65-4.96)\end{array}$ & $\begin{array}{l}1.18 \\
(0.57-2.45)\end{array}$ & $\begin{array}{l}1.78 \\
(0.62-5.08)\end{array}$ & $\begin{array}{l}1.19 \\
(0.56-2.50)\end{array}$ & 0.283 & 0.648 \\
\hline $\mathrm{CC}$ & 1 & 0.61 & 1 & 4.1 & 0 & 0 & $\begin{array}{l}8.06 \\
(0.48-134.8)\end{array}$ & - & $\begin{array}{l}6.98 \\
(0.41-118.82)\end{array}$ & - & 0.185 & - \\
\hline $\mathrm{CC}+\mathrm{CT}$ & 28 & 16.97 & 7 & 29.17 & 13 & 18.84 & $\begin{array}{l}2.02 \\
(0.76-5.31)\end{array}$ & $\begin{array}{l}1.13 \\
(0.54-2.35)\end{array}$ & $\begin{array}{l}2.01 \\
(0.74-5.45)\end{array}$ & $\begin{array}{l}1.16 \\
(0.55-2.42)\end{array}$ & 0.176 & 0.699 \\
\hline $\mathrm{TT}+\mathrm{CT}$ & 164 & 99.39 & 23 & 95.83 & 69 & 100 & $\begin{array}{l}1.00 \\
\text { (reference) }\end{array}$ & $\begin{array}{l}1.00 \\
\text { (reference) }\end{array}$ & $\begin{array}{l}1.00 \\
\text { (reference) }\end{array}$ & $\begin{array}{l}1.00 \\
\text { (reference) }\end{array}$ & & \\
\hline CC & 1 & 0.61 & 1 & 4.1 & 0 & 0 & $\begin{array}{l}7.13 \\
(0.43-117.96)\end{array}$ & - & $\begin{array}{l}6.39 \\
(0.38-108.06)\end{array}$ & - & 0.199 & - \\
\hline \multicolumn{13}{|l|}{ rs2069772 } \\
\hline $\mathrm{AA}$ & 134 & 81.21 & 19 & 79.17 & 56 & 81.16 & $\begin{array}{l}1.00 \\
\text { (reference) }\end{array}$ & $\begin{array}{l}1.00 \\
\text { (reference) }\end{array}$ & $\begin{array}{l}1.00 \\
\text { (reference) }\end{array}$ & $\begin{array}{l}1.00 \\
\text { (reference) }\end{array}$ & & \\
\hline AG & 30 & 18.18 & 41 & 16.67 & 12 & 17.39 & $\begin{array}{l}0.94 \\
(0.29-2.96)\end{array}$ & $\begin{array}{l}0.95 \\
(0.45-2.00)\end{array}$ & $\begin{array}{l}0.96 \\
(0.30-3.07)\end{array}$ & $\begin{array}{l}0.88 \\
(0.41-1.87)\end{array}$ & 0.953 & 0.742 \\
\hline GG & 1 & 0.61 & 1 & 4.17 & 1 & 1.45 & $\begin{array}{l}7.05 \\
(0.42-117.51)\end{array}$ & $\begin{array}{l}2.39 \\
(0.14-38.92)\end{array}$ & $\begin{array}{l}8.12 \\
(0.45-143.98)\end{array}$ & $\begin{array}{l}3.72 \\
(0.19-70.48)\end{array}$ & 0.157 & 0.386 \\
\hline $\mathrm{GG}+\mathrm{AG}$ & 31 & 18.79 & 5 & 20.83 & 13 & 18.84 & $\begin{array}{l}1.13 \\
(0.39-3.28)\end{array}$ & $\begin{array}{l}1.01 \\
(0.48-2.05)\end{array}$ & $\begin{array}{l}1.17 \\
(0.40-3.42)\end{array}$ & $\begin{array}{l}0.94 \\
(0.45-1.96)\end{array}$ & 0.761 & 0.889 \\
\hline$A A+A G$ & 164 & 99.39 & 23 & 95.83 & 68 & 98.55 & $\begin{array}{l}1.00 \\
\text { (reference) }\end{array}$ & $\begin{array}{l}1.00 \\
\text { (reference) }\end{array}$ & $\begin{array}{l}1.00 \\
\text { (reference) }\end{array}$ & $\begin{array}{l}1.00 \\
\text { (reference) }\end{array}$ & & \\
\hline GG & 1 & 0.61 & 1 & 4.17 & 1 & 1.45 & $\begin{array}{l}7.13 \\
(0.43-117.95)\end{array}$ & $\begin{array}{l}2.41 \\
(0.14-39.11)\end{array}$ & $\begin{array}{l}8.17 \\
(0.46-143.88)\end{array}$ & $\begin{array}{l}3.78 \\
(0.20-71.37)\end{array}$ & 0.155 & 0.372 \\
\hline \multicolumn{13}{|l|}{ rs2069762 } \\
\hline TT & 59 & 35.76 & 12 & 50.00 & 34 & 49.28 & $\begin{array}{l}1.00 \\
\text { (reference) }\end{array}$ & $\begin{array}{l}1.00 \\
\text { (reference) }\end{array}$ & $\begin{array}{l}1.00 \\
\text { (reference) }\end{array}$ & $\begin{array}{l}1.00 \\
\text { (reference) }\end{array}$ & & \\
\hline GT & 85 & 51.52 & 1 & 45.83 & 29 & 42.03 & $\begin{array}{l}0.63 \\
(0.26-1.54)\end{array}$ & $\begin{array}{l}0.59 \\
(0.32-1.07)\end{array}$ & $\begin{array}{l}0.65 \\
(0.27-1.60)\end{array}$ & $\begin{array}{l}0.57 \\
(0.31-1.05)\end{array}$ & 0.358 & 0.071 \\
\hline GG & 21 & 12.73 & 1 & 4.17 & 6 & 8.70 & $\begin{array}{l}0.23 \\
(0.03-1.91)\end{array}$ & $\begin{array}{l}0.49 \\
(0.18-1.35)\end{array}$ & $\begin{array}{l}0.22 \\
(0.02-1.83)\end{array}$ & $\begin{array}{l}0.56 \\
(0.20-1.54)\end{array}$ & 0.162 & 0.261 \\
\hline $\mathrm{GG}+\mathrm{GT}$ & 106 & 64.24 & 12 & 50.00 & 35 & 50.72 & $\begin{array}{l}0.55 \\
(0.23-1.32)\end{array}$ & $\begin{array}{l}0.57 \\
(0.32-1.01)\end{array}$ & $\begin{array}{l}0.56 \\
(0.23-1.34)\end{array}$ & $\begin{array}{l}0.56 \\
(0.32-1.01)\end{array}$ & 0.197 & 0.055 \\
\hline $\mathrm{TT}+\mathrm{GT}$ & 144 & 87.27 & 23 & 95.83 & 63 & 91.30 & $\begin{array}{l}1.00 \\
\text { (reference) }\end{array}$ & $\begin{array}{l}1.00 \\
\text { (reference) }\end{array}$ & $\begin{array}{l}1.00 \\
\text { (reference) }\end{array}$ & $\begin{array}{l}1.00 \\
\text { (reference) }\end{array}$ & & \\
\hline GG & 21 & 12.73 & 1 & 4.17 & 6 & 8.70 & $\begin{array}{l}0.29 \\
(0.03-2.32)\end{array}$ & $\begin{array}{l}0.65 \\
(0.25-1.69)\end{array}$ & $\begin{array}{l}0.27 \\
(0.03-2.17)\end{array}$ & $\begin{array}{l}0.74 \\
(0.28-1.96)\end{array}$ & 0.222 & 0.546 \\
\hline \multicolumn{13}{|l|}{ rs2227306 } \\
\hline $\mathrm{CC}$ & 63 & 38.18 & 6 & 25.00 & 31 & 44.93 & $\begin{array}{l}1.00 \\
\text { (reference) }\end{array}$ & $\begin{array}{l}1.00 \\
\text { (reference) }\end{array}$ & $\begin{array}{l}1.00 \\
\text { (reference) }\end{array}$ & $\begin{array}{l}1.00 \\
\text { (reference) }\end{array}$ & & \\
\hline $\mathrm{CT}$ & 82 & 49.70 & 15 & 62.50 & 29 & 42.03 & $\begin{array}{l}1.92 \\
(0.70-5.23)\end{array}$ & $\begin{array}{l}0.72 \\
(0.39-1.31)\end{array}$ & $\begin{array}{l}1.86 \\
(0.68-5.10)\end{array}$ & $\begin{array}{l}0.72 \\
(0.39-1.32)\end{array}$ & 0.228 & 0.286 \\
\hline TT & 20 & 12.12 & 3 & 12.50 & 9 & 13.04 & $\begin{array}{l}1.57 \\
(0.36-6.88)\end{array}$ & $\begin{array}{l}0.92 \\
(0.37-2.24)\end{array}$ & $\begin{array}{l}1.64 \\
(0.37-7.24)\end{array}$ & $\begin{array}{l}0.88 \\
(0.35-2.17)\end{array}$ & 0.512 & 0.778 \\
\hline $\mathrm{TT}+\mathrm{CT}$ & 102 & 61.82 & 18 & 75.00 & 38 & 55.07 & $\begin{array}{l}1.85 \\
(0.69-4.92)\end{array}$ & $\begin{array}{l}0.76 \\
(0.43-1.33)\end{array}$ & $\begin{array}{l}1.82 \\
(0.68-4.85)\end{array}$ & $\begin{array}{l}0.75 \\
(0.42-1.33)\end{array}$ & 0.232 & 0.327 \\
\hline $\mathrm{CC}+\mathrm{CT}$ & 145 & 87.88 & 21 & 87.50 & 60 & 86.96 & $\begin{array}{l}1.00 \\
\text { (reference) }\end{array}$ & $\begin{array}{l}1.00 \\
\text { (reference) }\end{array}$ & $\begin{array}{l}1.00 \\
\text { (reference) }\end{array}$ & $\begin{array}{l}1.00 \\
\text { (reference) }\end{array}$ & & \\
\hline TT & 20 & 12.12 & 31 & 12.50 & 9 & 13.04 & $\begin{array}{l}1.03 \\
(0.28-3.79)\end{array}$ & $\begin{array}{l}1.08 \\
(0.46-2.52)\end{array}$ & $\begin{array}{l}1.10 \\
(0.29-4.08)\end{array}$ & $\begin{array}{l}1.04 \\
(0.44-2.45)\end{array}$ & 0.883 & 0.922 \\
\hline \multicolumn{13}{|l|}{ rs 2227543} \\
\hline $\mathrm{CC}$ & 60 & 36.36 & 62 & 25.00 & 28 & 40.58 & $\begin{array}{l}1.00 \\
\text { (reference) }\end{array}$ & $\begin{array}{l}1.00 \\
\text { (reference) }\end{array}$ & $\begin{array}{l}1.00 \\
\text { (reference) }\end{array}$ & $\begin{array}{l}1.00 \\
\text { (reference) }\end{array}$ & & \\
\hline CT & 83 & 50.30 & 14 & 58.33 & 31 & 44.93 & $\begin{array}{l}1.68 \\
(0.61-4.64)\end{array}$ & $\begin{array}{l}0.80 \\
(0.43-1.47)\end{array}$ & $\begin{array}{l}1.62 \\
(0.58-4.49)\end{array}$ & $\begin{array}{l}0.81 \\
(0.43-1.51)\end{array}$ & 0.354 & 0.512 \\
\hline TT & 22 & 13.33 & 4 & 16.67 & 10 & 14.49 & $\begin{array}{l}1.82 \\
(0.47-7.05)\end{array}$ & $\begin{array}{l}0.97 \\
(0.40-2.33)\end{array}$ & $\begin{array}{l}1.90 \\
(0.48-7.47)\end{array}$ & $\begin{array}{l}0.92 \\
(0.38-2.24)\end{array}$ & 0.355 & 0.865 \\
\hline $\mathrm{TT}+\mathrm{CT}$ & 105 & 63.64 & 18 & 75.00 & 41 & 59.42 & $\begin{array}{l}1.71 \\
(0.65-4.55)\end{array}$ & $\begin{array}{l}0.83 \\
(0.47-1.48)\end{array}$ & $\begin{array}{l}1.68 \\
(0.62-4.48)\end{array}$ & $\begin{array}{l}0.83 \\
(0.46-1.50)\end{array}$ & 0.302 & 0.552 \\
\hline $\mathrm{CC}+\mathrm{CT}$ & 143 & 86.67 & 20 & 83.33 & 59 & 85.51 & $\begin{array}{l}1.00 \\
\text { (reference) }\end{array}$ & $\begin{array}{l}1.00 \\
\text { (reference) }\end{array}$ & $\begin{array}{l}1.00 \\
\text { (reference) }\end{array}$ & $\begin{array}{l}1.00 \\
\text { (reference) }\end{array}$ & & \\
\hline TT & 22 & 13.33 & 4 & 16.67 & 10 & 14.49 & $\begin{array}{l}1.30 \\
(0.41-4.16)\end{array}$ & $\begin{array}{l}1.10 \\
(0.49-2.47)\end{array}$ & $\begin{array}{l}1.40 \\
(0.43-4.54)\end{array}$ & $\begin{array}{l}1.03 \\
(0.45-2.35)\end{array}$ & 0.575 & 0.930 \\
\hline
\end{tabular}

Furthermore, we carried out stratified analysis of association between rs2069762 polymorphism and acquired AA risk. The analysis results were showed in Table 4. A decreased risk was observed in adults ( $>16$ years old) with GT and GT/GG genotypes.

According to the disease severity, acquired AA could be further subdivided into three groups: vSAA, SAA and NSAA. Since our sample size was small and eight patients lack the information of disease severity, vSAA patients were so little that we combined the vSAA and SAA together to analysis. The distribution of five selected SNPs genotypes were compared 
Fig. 1. Transcription factor binding sites showed below the DNA sequence were predicted by TFSearch while it was nucleotide $\mathrm{T}$ at rs2069762. Full score was 100 , the higher the score the greater the chance.

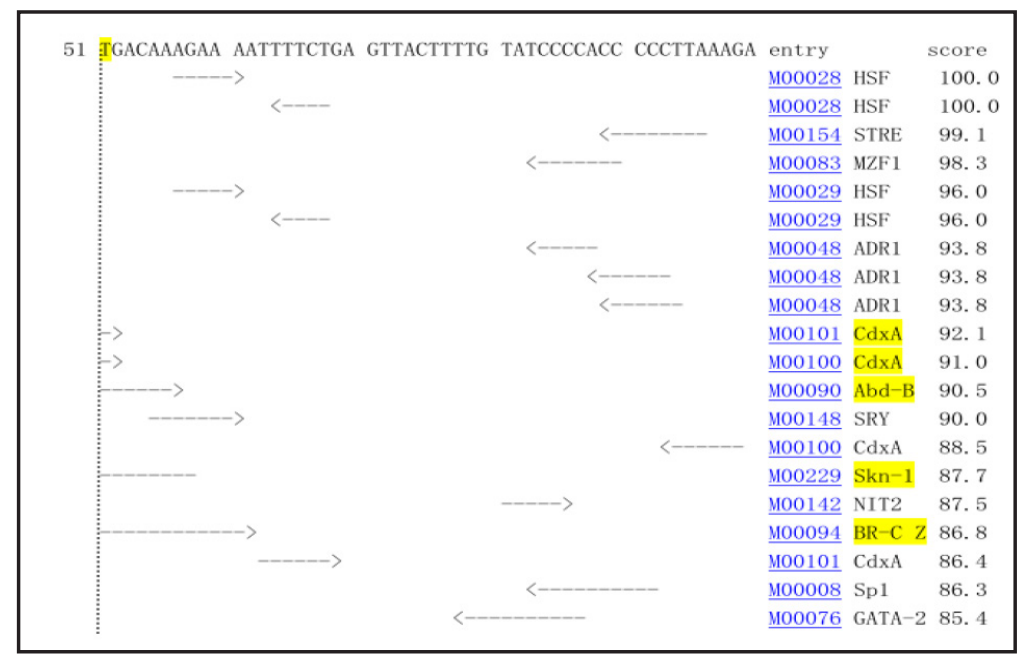

Fig. 2. No transcription factor binding site was found when it was nucleotide $G$ at rs2069762 predicted by TFSearch.

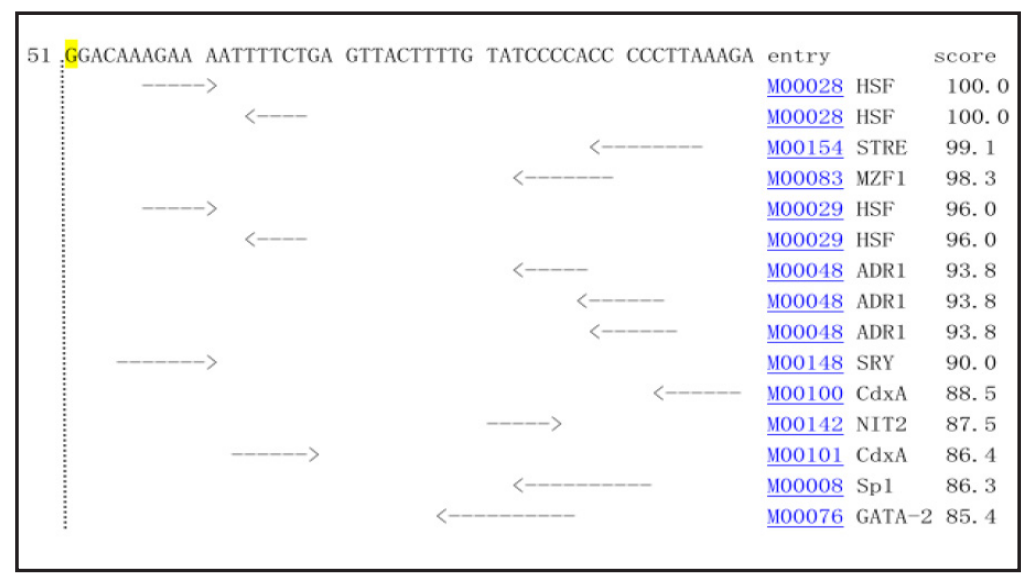

between each of these two groups and the controls, respectively. As showed in Table 5, no statistical difference was found in our study.

\section{Functional information}

Rs2069762 was found located in a transcription factor binding region from SNPinfo. We further predict the potential transcription factor binding sites in rs2069762 by F-SNP. Data shown at this web site was come from the prediction tools of TFSearch. Transcription factor binding sites including CdxA, Abd-B, Skn-1 and BR-C Z were predicted to be exist when nucleotide $\mathrm{T}$ located in rs2069762 (Fig. 1). However, no TFBS was found while nucleotide G located in this polymorphic loci (Fig. 2). It indicated that rs2069762 changed the transcriptional regulation.

Moreover, previous researchers had explored whether rs2069762 could affect the transcriptional activity. They conducted experiments of plasmid construction, transfection and luciferase assays. Results showed statistical differences [15]. These information reflected that rs2069762 could ulteriorly influence expression or function of IL-2.

\section{Discussion}

According to Neal S.Young's report in 2008, the annual incidence of acquired aplastic anemia in China was about 7.4 per million, approximately 3-fold higher than that in Europe [24]. Though the etiology of acquired AA has some correlations with immune dysregulation, viral infections, drug and chemical exposure, the exact pathogenesis still remains idiopathic 
and elusive. Nowadays, scholars mostly agree that acquired AA is an autoimmune disease. About two-thirds of acquired aplastic anemia patients could recover their hematopoietic function after immunosuppressive therapies (IST), serving as an important evidence for the theory that acquired AA is an autoimmune disease [24]. In most cases, the active destruction of hematopoietic cells by effector T lymphocytes is a key factor leading to the occurrence of acquired aplastic anemia. It was found that abnormal activation of Th1 T-cell would make patients' $\mathrm{T}$ cells immoderately produce hematopoietic negative regulatory factors, which inhibit the proliferation of HSPC and weaken the hematopoietic regulation mechanism [5].

As an autocrine product from antigen-stimulated T cells, IL-2 induces the production of proinflammatory cytokines like IFN- $\gamma$ and IL-4, which play a crucial role in proliferation and expansion of both antigen-specific clones of CD4+ and CD8+ T cells [25]. Besides, IL-2 can control and prevent autoimmunity by stimulating T regulatory (CD4+CD25+) cells [26]. IL-8, a member of the chemokine family, can suppress the proliferation of primitive myeloid cells by transducing an inhibitory signal via receptor-ligand-mediated mechanism $[27,28]$. Thus, these two cytokines both have subtle effects on the development of acquired aplastic anemia.

SNP is caused by the variation of base sequence at the genome level. The changed genotype may have impacts on DNA transcription or protein structure that could affect receptor binding or protein concentration [29]. Then the biological properties would be affected. In this case, SNP could cause the susceptibility of disease.

As far as we know, this is the first time to investigate the correlation between these five SNPs (rs11575812, rs2069772 and rs2069762 of IL-2, rs2227306 and rs2227543 of IL-8) and the risk of acquired AA in Chinese Han population. Rs2069762, located in a 5' flanking region of IL2, is statistically significant in our study. At the same time, the functional information we found showed that it changed the transcriptional regulation. It was also reported that rs2069762 had an impact on expression of IL-2 [30]. In this case, IL-2 may further negatively affect the $\mathrm{T}$ lymphocyte mediated immune response, contributing to the occurrence of acquired aplastic anemia. Moreover, it was also discovered that rs2069762 $\mathrm{T}$ to $\mathrm{G}$ substitution was associated with the development of other autoimmune diseases, such as multiple sclerosis (MS) in Spainish population [15] and psoriasis in the Northeastern Chinese Han population [31]. Consistently, data in table 4 showed a decreased risk in adults (>16 years old) with GT and GT/GG genotypes. Since our sample size was small, homozygous mutations were too few, possibly making the result biased. In that case, the statistical result of heterozygote might become much more reliable. Therefore, it could be assumed that the rs2069762 T > G mutation might reduce the risk of acquired aplastic anemia in a Chinese population. This conclusion needs to be validated with a larger sample size.

Although according to the results of our study, rs11575812, rs2069772, rs2227306 and rs2227543 might not have association with the occurrence of acquired AA, they have association with other diseases. It was found that mutation in rs11575812 could be associated with SLE risk in a Tunisian population [25]. Henneman et al. reported rs2069772 variant might contribute to the expression of Cow's milk allergy in Dutch children [32]. Polymorphisms of rs2227306 and rs2227543 might confer the development and progression of childhood IgA nephropathy [33]. In addition, unfortunately, no meaningful results were found between the two groups when stratified by the disease severity including NSAA, SAA and vSAA (table 5). However, due to our relatively small sample size, it is hard to draw the conclusion that these five SNPs have no connection with the disease severity; a much larger sample size are needed to consolidate the conclusion.

In conclusion, results in this study suggest that $\mathrm{rs} 2069762 \mathrm{~T}>\mathrm{G}$ mutation may be associated with a reduced risk for acquired AA in a Chinese population. Although the sample size is relatively small, our findings have provided direction for further research with a much larger sample size. Moreover, based on the results of our study, further epidemiological studies in a larger sample size and functional evaluations could be carried out. 


\section{Cellular Physiology Cell Physiol Biochem 2017;41:1199-1207 \begin{tabular}{l|l} 
and Biochemistry POI: 10.1159/000464381 & $\begin{array}{l}\text { (c) } 2017 \text { The Author(s). Published by S. Karger AG, Basel } \\
\text { www.karger.com/cpb }\end{array}$
\end{tabular} \\ \begin{tabular}{l|l} 
Published online: March 06, $2017 \quad$ www.karger.com/cpb \\
\hline Zhang et al: IL-2 and IL-8 Polymorphisms with Acquired AA
\end{tabular}}

\section{Acknowledgement}

This research was supported by the National Natural Science Foundation of China (81602913,81670155), the Natural Science Foundation of Jiangsu Province (BK20150085), Nanjing Medical Science and Technology Development Project (YKK15140), Nanjing medical university (2014NJMU156) and Nanjing Science and Technology Development Foundation (201503043).

\section{Disclosure Statement}

None declared.

\section{References}

1 Young NS, Scheinberg P, Calado RT: Aplastic anemia. Curr Opin Hematol 2008;15:162-168.

-2 Kook H, Chung NG, Kang HJ, Im HJ: Acquired aplastic anemia in Korean children: treatment guidelines from the Bone Marrow Failure Committee of the Korean Society of Pediatric Hematology Oncology. Int J Hematol 2016;103:380-386.

- 3 Betensky M, Babushok D, Roth JJ, Mason PJ, Biegel JA, Busse TM, Li Y, Lind C, Papazoglou A, Monos D, Podsakoff G, Bessler M, Olson TS: Clonal evolution and clinical significance of copy number neutral loss of heterozygosity of chromosome arm 6p in acquired aplastic anemia. Cancer Genet 2016;209:1-10.

4 Ge M, Yu W, Shi J, Zhang J, Wang M, Feng S, Li X, Shao Y, Zheng Y: TRAF1/C5 rs10818488 polymorphism is not a genetic risk factor for acquired aplastic anemia in a Chinese population. Hum Immunol 2015;76:1921.

5 Solomou EE, Keyvanfar K, Young NS: T-bet, a Th1 transcription factor, is up-regulated in T cells from patients with aplastic anemia. Blood 2006;107:3983-3991.

6 Tripathy NK, Nityanand S, Vibhuti: Bone marrow and blood plasma levels of IL-8 in aplastic anemia and their relationship with disease severity. Am J Hematol 2005;79:240-242.

7 Nelson BH: IL-2, Regulatory T Cells, and Tolerance. J Immunol 2004;172:3983-3988.

8 Malek TR: The biology of interleukin-2. Annu Rev Immunol 2008;26:453-479.

-9 Hoyer KK, Dooms H, Barron L, Abbas AK: Interleukin-2 in the development and control of inflammatory disease. Immunol Rev 2008;226:19-28.

10 Singh PK, Chandra G, Bogra J, Gupta R, Kumar V, Hussain SR, Jain A, Mahdi AA, Ahmad MK: Association of Genetic Polymorphism in the Interleukin-8 Gene with Risk of Oral Cancer and Its Correlation with Pain. Biochem Genet 2016;54:95-106.

-11 Giannakoulas NC, Karakantza M, Theodorou GL, Pagoni M, Galanopoulos A, Kakagianni T, KouraklisSymeonidis A, Matsouka P, Maniatis A, Zoumbos NC: Clinical relevance of balance between type 1 and type 2 immune responses of lymphocyte subpopulations in aplastic anaemia patients. Br J Haematol 2004;124:97-105.

12 Fichna M, Zurawek M, Fichna P, Ziolkowska-Suchanek I, Januszkiewicz D, Nowak J: Polymorphic variant at the IL2 region is associated with type 1 diabetes and may affect serum levels of interleukin-2. Mol Biol Rep 2013;40:6957-6963.

13 Zhernakova A, Alizadeh BZ, Bevova M, van Leeuwen MA, Coenen MJ, Franke B, Franke L, Posthumus MD, van Heel DA, van der Steege G, Radstake TR, Barrera P, Roep BO, Koeleman BP, Wijmenga C: Novel association in chromosome 4q27 region with rheumatoid arthritis and confirmation of type 1 diabetes point to a general risk locus for autoimmune diseases. Am J Hum Genet 2007;81:1284-1288.

14 Albers HM, Kurreeman FA, Stoeken-Rijsbergen G, Brinkman DM, Kamphuis SS, van Rossum MA, Girschick HJ, Wouters C, Saurenmann RK, Hoppenreijs E, Slagboom P, Houwing-Duistermaat JJ, Verduijn W, Huizinga TW, Ten Cate R, Toes RE, Schilham MW: Association of the autoimmunity locus 4q27 with juvenile idiopathic arthritis. Arthritis Rheum 2009;60:901-904. 


\section{Cellular Physiology Cell Physiol Biochem 2017;41:1199-1207 \begin{tabular}{ll|l} 
DOI: 10.1159/000464381 and Biochemistry & $\begin{array}{l}\text { O 2017 The Author(s). Published by S. Karger AG, Basel } \\
\text { www.karger.com/cpb }\end{array}$
\end{tabular}

15 Matesanz F, Fedetz M, Leyva L, Delgado C, Fernandez O, Alcina A: Effects of the multiple sclerosis associated -330 promoter polymorphism in IL2 allelic expression. J Neuroimmunol 2004;148:212-217.

16 Warren RB, Smith RL, Flynn E, Bowes J, Eyre S, Worthington J, Barton A, Griffiths CE: A systematic investigation of confirmed autoimmune loci in early-onset psoriasis reveals an association with IL2/IL21. Br J Dermatol 2011;164:660-664.

17 Festen EA, Goyette P, Scott R, Annese V, Zhernakova A, Lian J, Lefebvre C, Brant SR, Cho JH, Silverberg MS, Taylor KD, de Jong DJ, Stokkers PC, McGovern D, Palmieri O, Achkar JP, Xavier RJ, Daly MJ, Duerr RH, Wijmenga C, Weersma RK, Rioux JD: Genetic variants in the region harbouring IL2/IL21 associated with ulcerative colitis. Gut 2009;58:799-804.

-18 Maiti AK, Kim-Howard X, Viswanathan P, Guillen L, Rojas-Villarraga A, Deshmukh H, Direskeneli H, Saruhan-Direskeneli G, Canas C, Tobon GJ, Sawalha AH, Chernavsky AC, Anaya JM, Nath SK: Confirmation of an association between rs6822844 at the Il2-Il21 region and multiple autoimmune diseases: evidence of a general susceptibility locus. Arthritis Rheum 2010;62:323-329.

-19 Sawalha AH, Kaufman KM, Kelly JA, Adler AJ, Aberle T, Kilpatrick J, Wakeland EK, Li QZ, Wandstrat AE, Karp DR, James JA, Merrill JT, Lipsky P, Harley JB: Genetic association of interleukin-21 polymorphisms with systemic lupus erythematosus. Ann Rheum Dis 2008;67:458-461.

20 Chen Y, Yang Y, Liu S, Zhu S, Jiang H, Ding J: Association between interleukin 8 -251 A/T and +781 $\mathrm{C} / \mathrm{T}$ polymorphisms and osteosarcoma risk in Chinese population: a case-control study. Tumour Biol 2016;37:6191-6196.

21 Wang Z, Wang C, Zhao Z, Liu F, Guan X, Lin X, Zhang L: Association between -251A>T polymorphism in the interleukin-8 gene and oral cancer risk: a meta-analysis. Gene 2013;522:168-176.

-22 Xue H, Liu J, Lin B, Wang Z, Sun J, Huang G: A meta-analysis of interleukin-8 -251 promoter polymorphism associated with gastric cancer risk. PLoS One 2012;7:e28083.

-23 He Y, Liang X, Wu X, Meng C, Wu B, Fu D, Jin S, Yang S, Wang H: Association between interleukin 8 -251 A/T and $+781 \mathrm{C} / \mathrm{T}$ polymorphisms and osteoarthritis risk. Immunol Lett 2014;162:207-211.

24 Young NS, Kaufman DW: The epidemiology of acquired aplastic anemia. Haematologica 2008;93:489-492.

25 Bouzid D, Fourati H, Amouri A, Marques I, Abida O, Tahri N, Penha-Goncalves C, Masmoudi H: Autoimmune diseases association study with the KIAA1109-IL2-IL21 region in a Tunisian population. Mol Biol Rep 2014;41:7133-7139.

26 Chistiakov DA, Voronova NV, Chistiakov PA: The crucial role of IL-2/IL-2RA-mediated immune regulation in the pathogenesis of type 1 diabetes, an evidence coming from genetic and animal model studies. Immunol Lett 2008;118:1-5.

27 Broxmeyer HE, Cooper S, Cacalano G, Hague NL, Bailish E, Moore MW: Involvement of Interleukin (IL) 8 receptor in negative regulation of myeloid progenitor cells in vivo: evidence from mice lacking the murine IL-8 receptor homologue. J Exp Med 1996;184:1825-1832.

28 Sanchez X, Suetomi K, Cousins-Hodges B, Horton JK, Navarro J: CXC chemokines suppress proliferation of myeloid progenitor cells by activation of the CXC chemokine receptor 2. J Immunol 1998;160:906-910.

-29 Hoffmann SC, Stanley EM, Darrin Cox E, Craighead N, DiMercurio BS, Koziol DE, Harlan DM, Kirk AD, Blair PJ: Association of cytokine polymorphic inheritance and in vitro cytokine production in anti-CD3/CD28stimulated peripheral blood lymphocytes. Transplantation 2001;72:1444-1450.

-30 Zhao H, Wang R: IL-2 -330T/G polymorphism and cancer risk: a meta-analysis. Onco Targets Ther 2015;8:1753-1760.

-31 Zhou J, Wang L, Wang F, Xu P, Li Y, Bai B, Dang L, Sun D: 4q27 as a psoriasis susceptibility locus in the Northeastern Chinese Han population. Tissue Antigens 2015;85:15-19.

-32 Henneman P, Petrus NC, Venema A, van Sinderen F, van der Lip K, Hennekam RC, Mannens M, Sprikkelman AB: Genetic susceptibility for cow's milk allergy in Dutch children: the start of the allergic march? Clin Transl Allergy 2015;6:7.

33 Suh JS, Hahn WH, Cho BS: Polymorphisms of CXCL8 and its receptor CXCR2 contribute to the development and progression of childhood IgA nephropathy. J Interferon Cytokine Res 2011;31:309-315. 